\title{
Hypoglycemic Effects of Morus alba Leaf Extract on Postprandial Glucose and Insulin Levels in Patients with Type 2 Diabetes Treated with Sulfonylurea Hypoglycemic Agents
}

Sadako Nakamura, Michiru Hashiguchi, Yoshihiko Yamaguchi and Tsuneyuki Oku*

Graduate School of Human Health Science, University of Nagasaki Siebold, 1-1-1 Manabino, Nagayo, Nagasaki 8512195, Japan

\begin{abstract}
Phytochemical extracts with a-glucosidase inhibitory activity are widely used in processed foods with hypoglycemic effect. However the interactions between these phytochemical extracts and prescribed medicines have not yet been investigated. The leaf extract of Morus alba (LEM) shows the competitive inhibition to a-glucosidase. This singleblinded, placebo-controlled study investigated the effects of LEM on postprandial glucose and insulin levels in type 2 diabetes patients treated with or without sulfonylurea hypoglycemic agents (SU). Blood was collected from patients and healthy subjects at the indicated times after the ingestion of jelly containing LEM. A hydrogen breath test was performed simultaneously in healthy subjects to detect undigested sucrose in the jelly, which is fermented by intestinal microbes to produce hydrogen. Postprandial elevations in glucose and insulin levels were significantly suppressed in patients with and without SU treatment after ingestion of jelly containing LEM, compared to placebo jelly $(p<0.05)$. Elevations in glucose and insulin levels were suppressed and the excretion of breath hydrogen gas was markedly increased in healthy subjects after ingestion of jelly containing LEM. These results suggest that LEM can suppress the postprandial elevation of glucose and insulin independent of SU treatment. These results could help to improve food processing for diet therapy in diabetes.
\end{abstract}

Keywords: Postprandial glucose; Insulin; a-glucosedase inhibitor; Morus alba; Type 2 diabetes; SU treatment

\section{Introduction}

Phytochemical extracts with $\alpha$-glucosidase inhibitory activity have been shown to suppress postprandial increases in glucose and insulin levels. They are widely used in processed foods that have been developed to prevent lifestyle-related diseases. The leaf extract of Morus alba (LEM) also has a suppressive effect on postprandial glucose [1-3]. However, the interactions between LEM and prescribed medicines, and the resulting effects on postprandial glucose and insulin, have not yet been investigated.

LEM markedly suppressed postprandial glucose and insulin in healthy subjects and normal rats [1-3], and Mudra et al. reported that mulberry suppressed blood glucose after sucrose administration in diabetic subjects [4]. Furthermore, the appearance of polyuria and the elevation of fasting blood glucose and insulin were delayed by the daily intake of LEM in diabetic mice [5]. LEM has also been reported to have other physiological effects, including the inhibition of low-density lipoprotein oxidation in vitro [6], and the attenuation of atherosclerotic lesion development in mice [7]. We previously clarified that confections containing LEM suppressed postprandial elevations of glucose and insulin in healthy subjects [8]. Competitive inhibition by LEM for a-glucosidases has been identified as the mechanism responsible for this suppression [1]. However, the range of insulin concentrations and the variety of medical treatments mean that more detailed studies are needed to evaluate the suppressive effects of LEM on postprandial glucose and insulin in diabetic patients.

Diabetes mellitus is an irreversible disease, but it is important that patients maintain adequate blood glucose and insulin levels $[9,10]$, to help prevent complications and the progression of diabetes to more serious stages. The concentrations of blood glucose and insulin in diabetic patients vary widely from patient to patient, and the medical treatment differs from case to case. Improvements in lifestyle are commonly required, and confections are usually strictly prohibited. However, strict diet therapy may have adverse effects, such as transitory hyperorexia and depression [11-13]. To help address these problems, we developed a jelly containing LEM that had suppressive effects on the increments in postprandial glucose and insulin secretion.

LEM, which includes the D-glucose analogs 1-deoxynojirimycin and its derivatives, [14], has an inhibitory effect on $\alpha$-glucosidases [1$4,8]$. The current study subjects were limited to patients with type 2 diabetes who were receiving sulfonylurea hypoglycemic agents (SU), but not $\alpha$-glucosidase inhibitors, and untreated patients.

SU stimulates the secretion of insulin for the patients with insulinindependent and are used as first-line treatments for type 2 diabetes, but recently SU added glucagon-like peptide-1 are also used in Japan [15]. SU binds to the SU-receptor (SUR) in pancreas beta-cell which consist ATP-dependent potassium channel. On the other hand, SU exhausts the beta-cell due to the oxidation stress, however, Nyback-Nakell et al. do not support that SU is harmful to beta-cell function [16], and a small dose of insulin effectively prevent beta-cell failure caused by SUtreatment in type 1 diabetes [17]. The intake of carbohydrate between meals causes higher concentration of blood glucose and then vascular

*Corresponding author: Tsuneyuki Oku, PhD, 1-1-1 Manabino, Nagayo, Nagasaki 851-2195, Japan, Tel: +81-95-813-5211; E-mail: okutsune@sun.ac.jp

Received November 01, 2011; Accepted December 16, 2011; Published December 21, 2011

Citation: Nakamura S, Hashiguchi M, Yamaguchi Y, Oku T (2011) Hypoglycemic Effects of Morus alba Leaf Extract on Postprandial Glucose and Insulin Levels in Patients with Type 2 Diabetes Treated with Sulfonylurea Hypoglycemic Agents. J Diabetes Metab 2:158. doi:10.4172/2155-6156.1000158

Copyright: (c) 2011 Nakamura S, et al. This is an open-access article distributed under the terms of the Creative Commons Attribution License, which permits unrestricted use, distribution, and reproduction in any medium, provided the original author and source are credited. 
Citation: Nakamura S, Hashiguchi M, Yamaguchi Y, Oku T (2011) Hypoglycemic Effects of Morus alba Leaf Extract on Postprandial Glucose and Insulin Levels in Patients with Type 2 Diabetes Treated with Sulfonylurea Hypoglycemic Agents. J Diabetes Metab 2:158. doi:10.4172/21556156.1000158

Page 2 of 5

system is exposed oxidative stress. Anti-oxidative effects are very important for diabetic patients. LEM does not directly stimulate insulin secretion, and suppress the glucose elevation. Furthermore, it improves anti-oxidative and anti-glycation activities in streptozotocin-induced chronic diabetic rat [18], also has the protective effects on vascular system in rats fed atherogenic diet [19], and on ocular functions in pups from diabetic mother rat [20]. So, the combinational use of SU-treatment and LEM is associated with the protection of systemic oxidative stress.

Suppression of the postprandial glucose increment by LEM in healthy human subjects has been shown to result in the excretion of hydrogen gas in the breath $[4,8]$. This indicates fermentation by intestinal microbes of carbohydrates that have escaped digestion in the small intestine and been transferred to the large intestine $[21,22]$. Hydrogen excretion was also observed when an a-glucosidase inhibitor, such as acarbose or miglitol, was administered to patients with available carbohydrates [23-25]. The available energy of nondigestible saccharides has been estimated at $2 \mathrm{kcal} / \mathrm{g}(8.36 \mathrm{~kJ} / \mathrm{g})$ according to the Health Promotion Act in Japan [26,27], which is lower than that of digestible carbohydrates (approximately 4 kcal, $16.72 \mathrm{~kJ}$ ). LEM would therefore be expected to have an energy-reducing as well as hypoglycemic effect.

In this study, we designed a jelly containing LEM and investigated its effects on postprandial glucose and insulin levels in patients with type 2 diabetes treated with or without SU medicine and in healthy subjects, and compared the results with those obtained with a placebo jelly. We also investigated the potential energy-reducing effect of LEM based on the excretion of breath hydrogen gas. The results of this study could help to maintain the health and promote the quality of life in patients with type 2 diabetes mellitus.

\section{Subjects and Methods}

\section{Subjects}

Ten patients ( 5 male, 5 female) with type 2 diabetes mellitus participated in this study. Their diagnoses were carefully examined by the medical doctor. Five patients were receiving SUs, and no patients were receiving $\alpha$-glucosidase inhibitors. Ten healthy subjects ( 4 male, 6 female), with no history of diabetes or carbohydrate malabsorption, and who had received no antibiotics or laxatives for at least 2 weeks prior to the experiment, participated as controls. Each subject gave informed written consent to participate in this study. The characteristics of the subjects are shown in (Table 1).

\section{Test substances}

Powdered LEM: The powdered LEM contained $0.77 \%$ 1-deoxynojirimycin equivalents and trace amounts of other 1-deoxynojirimycin derivatives (Toyotama Healthy Food Co., Ltd., Tokyo, Japan). The safety of LEM has already been determined by Miyazawa et al. [28]. Digestible dextrin was added to the LEM to facilitate mixing and dissolution during food processing. LEM is khakicolored with the flavor of green grass.

Preparation of the test substances: The contents of the LEM and placebo jellies are shown in (Table 2). The amounts of sucrose and LEM in the test substances were calculated based on our previous study, and a ratio of sucrose to LEM of 10:1 was considered to be adequate [8]. The jellies contained $30.0 \mathrm{~g}$ of sucrose, $20.0 \mathrm{~g}$ of orange puree to mask the

\begin{tabular}{|l|c|c|}
\hline & Type 2 diabetic patients & Healthy subjects \\
\hline Age $(\mathrm{y})$ & $62.4 \pm 12.5$ & $23.6 \pm 2.1$ \\
Height $(\mathrm{cm})$ & $157.5 \pm 9.1$ & $156.8 \pm 5.3$ \\
Weight $(\mathrm{kg})$ & $60.7 \pm 8.9$ & $48.8 \pm 4.2$ \\
BMI $\left(\mathrm{kg} / \mathrm{m}^{2}\right)$ & $24.4 \pm 2.0$ & $20.1 \pm 1.8$ \\
Fasting blood glucose & $127.5 \pm 21.1$ & $84.0 \pm 3.6$ \\
(mg/dl) & & \\
HOMA-IR & $4.3 \pm 2.0$ & $1.5 \pm 0.7$ \\
\hline
\end{tabular}

Numbers: patients: 10 (male, 5; female, 5), healthy subjects: 10 (male, 4; Female 6) Values were expressed as means and SD.

Table 1: Characteristics of the subjects.

\begin{tabular}{|l|c|c|}
\hline & Jelly containing LEM & Placebo jelly \\
\hline Sugar & 30.0 & 30.0 \\
Gelatin & 1.7 & 1.7 \\
Orange puree & 20.0 & 20.0 \\
LEM & 3.3 & 0 \\
Dextrin & 0 & 3.3 \\
Water & 46.0 & 46.0 \\
\hline
\end{tabular}

Unit: g/portion $(80 \mathrm{~g})$. LEM was not added in placebo jelly.

Table 2: Ingredient contents of jelly containing LEM.

flavor and color of LEM, and $1.7 \mathrm{~g}$ of gelatin. A total of $3.3 \mathrm{~g}$ of LEM was added to the test jelly ( $254 \mu \mathrm{g}$ of deoxynojirimycin), and no LEM was added to the placebo jelly. The jellies were made by a competent confectioner, according to our directions and recipe, packed in plastic containers with lids, and transported under air-conditioning at a temperature below $-10^{\circ} \mathrm{C}$. On the experimental day, they were thawed in a refrigerator.

Experimental protocol: This study was designed as a singleblinded, placebo-controlled study, and was carried out as described in our previous study $[8,29,30]$, under the direction of a medical doctor.

Concentrations of glucose and insulin in patients and healthy subjects were compared between subjects who ate LEM and those who ate placebo jellies. The excretion of breath hydrogen gas was only measured in the healthy subjects, because it was considered to be too invasive to continue to collect end-expiratory samples in patients for 8 $\mathrm{h}$ after the ingestion of the test substance.

On the day before the experiment, the subjects finished their meals before 20:00 and fasted overnight, except for drinking water or Japanese green tea. On the day of the experiment, the subjects refrained from using any medication after waking, and came to the hospital at 08:00. Blood pressure and pulse were measured after resting, and a medical check was performed. Blood was then collected from both patients and healthy subjects to measure basal levels, with simultaneous collection of end-expiratory gas in the healthy subjects. After the first collection of blood and expiratory gas, subjects ingested the jelly with $150 \mathrm{ml}$ of drinking water. Blood was then collected at 30-min intervals for 2 $\mathrm{h}$ in the patients, and for $3 \mathrm{~h}$ in the healthy subjects. In the healthy subjects alone, $750-\mathrm{ml}$ samples of end-expiratory gas were collected at 1 -h intervals for $8 \mathrm{~h}$ after ingestion, using a special collection bag with a valve to stop backward flow (Quintron Instruments Co., Ltd., US) after the removal of dead-space gas.

The subjects were required to sit on a chair and were prohibited from smoking, sleeping, or exercising with hyperventilation until the final collection of blood and expiratory gas had been performed. No eating or drinking, except water, was allowed during the experiment. After blood collection, the healthy subjects ingested an experimental meal consisting of soft cookies and tea, which would not cause hydrogen gas excretion. 
Citation: Nakamura S, Hashiguchi M, Yamaguchi Y, Oku T (2011) Hypoglycemic Effects of Morus alba Leaf Extract on Postprandial Glucose and Insulin Levels in Patients with Type 2 Diabetes Treated with Sulfonylurea Hypoglycemic Agents. J Diabetes Metab 2:158. doi:10.4172/21556156.1000158

Blood glucose and insulin assays and hydrogen gas analysis: All blood samples were centrifuged at 3,000 $\mathrm{g}$ for $15 \mathrm{~min}$ at room temperature to obtain plasma. Plasma glucose concentrations were measured in duplicate by Trinder's method using glucose oxidase [31], and plasma insulin concentrations were measured by enzyme immunosorbent assay using an insulin assay kit [32] (Morinaga Seikagaku Corp., Kanagawa, Japan).

Twenty-five milliliters of end-expiratory gas was sucked into a plastic syringe and loaded into a simple gas chromatograph (Breath Gas Analyzer BGA1000D, Laboratory for Expiration Biochemistry Nourishment Metabolism Co., Ltd., Nara, Japan) to measure the hydrogen and methane gas concentrations.

Calculations and statistical analysis: Plasma glucose and insulin concentrations, and breath hydrogen gas excretion were calculated as means \pm standard deviations (SD) and tested for normal distributions. There were no significant differences between males and females in either the patients or the healthy subjects. To evaluate the suppressive effects of LEM jelly on the elevations of glucose and insulin, their levels at each time point were compared with those obtained using the placebo jelly. $\mathrm{P}$ values less than 0.05 obtained by two-sided analysis were considered to be significant using paired Student's $t$-tests by SPSS for Windows, Japan, version 15.0 (SPSS Inc., Tokyo, Japan).

Ethics: The study protocol was approved by the appropriate committees of University of Nagasaki Siebold, Japan and Miharadai Hospital, Japan. All experiments were carried out in the hospital and the Laboratory of Public Health Nutrition in University of Nagasaki Siebold.

\section{Results}

Effects of LEM jelly on postprandial glucose and insulin responses in patients with type 2 diabetes mellitus

Jelly containing LEM significantly suppressed the postprandial increases in blood glucose and insulin in patients treated with SU and in untreated patients, compared to placebo jelly (both $p<0.05$, Figure 1). Although insulin secretion was significantly suppressed by LEM jelly in patients both with and without SU treatment $(p<0.05)$, the insulin secretion profiles following the ingestion of placebo jelly differed, depending on whether patients were receiving SU. Figure 2 shows the elevations of postprandial glucose and insulin levels in all the patients. The mean fasting blood glucose level was $125 \pm 21 \mathrm{mg} / \mathrm{dl}$. The increase in blood glucose was significantly suppressed by ingestion of jelly containing $3.3 \mathrm{~g}$ of LEM $(148 \pm 29 \mathrm{mg} / \mathrm{dl})$, compared to ingestion of placebo jelly $(209 \pm 28 \mathrm{mg} / \mathrm{dl})$ at $30 \mathrm{~min}(p<0.05)$. The peak of glucose occurred at $60 \mathrm{~min}$ after ingestion $(160 \pm 27 \mathrm{mg} / \mathrm{dl})$. The postprandial insulin elevation was also significantly suppressed at $30 \mathrm{~min}$ after the ingestion of LEM jelly, compared to the ingestion of placebo jelly, but the inter-individual variation was large.

\section{Effects of LEM jelly on elevations of blood glucose and insulin in healthy subjects}

Figure 3 shows the postprandial glucose and insulin levels after the ingestion of LEM and placebo jellies in healthy subjects. The blood glucose increment at $30 \mathrm{~min}$ after the ingestion of LEM jelly was $97 \mathrm{mg} /$ $\mathrm{dl}$, which was significantly lower than that after ingestion of the placebo jelly $(125 \mathrm{mg} / \mathrm{ml}, p<0.05)$. Insulin secretion was also significantly
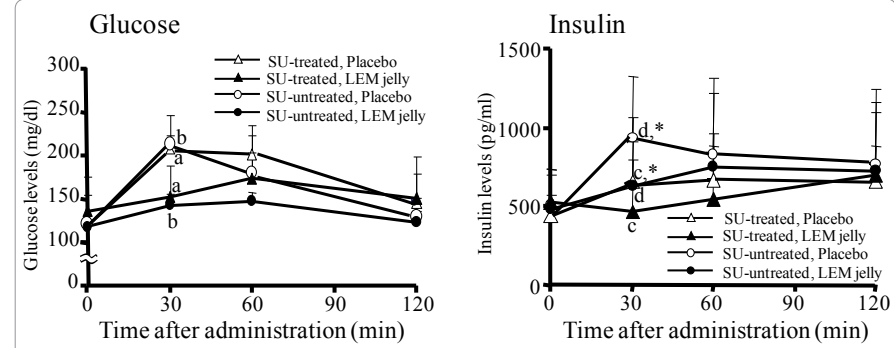

Figure 1: Effects of LEM jelly on postprandial glucose and insulin levels in patients with type 2 diabetes treated with or without SU. Data were expressed as means \pm SD (SU-treated, $n=5$; SU-untreated, $n=5$ ). a, c: Postprandial glucose and insulin levels were significantly suppressed by ingestion of jelly containing LEM, compared to the ingestion of placebo jelly in patients receiving $\mathrm{SU} ; p<0.05$ by paired Student's $t$-test. b, d: Significant differences were also observed in the postprandial glucose and insulin responses in patients not treated with SU; $p<0.05$ by paired Student's $t$-test. *There was a significant difference in insulin levels between SU-treated and SU-untreated patients after ingestion of placebo jelly; $p<0.05$ by Student's $t$-test.
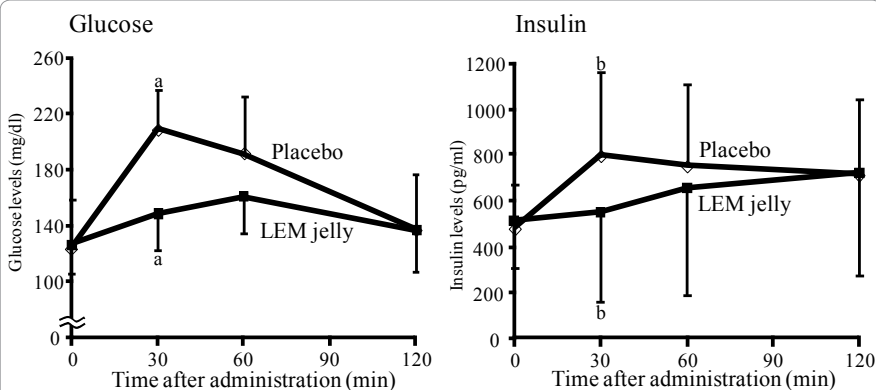

Figure 2: Effects of LEM jelly on postprandial glucose and insulin levels in type 2 diabetic patients. Data were expressed as means $\pm S D(n=10)$. a, b: Postprandial glucose and insulin levels were significantly suppressed by the ingestion of jelly containing LEM, compared to the ingestion of placebo jelly; $p<0.05$ by paired Student's $t$-test.

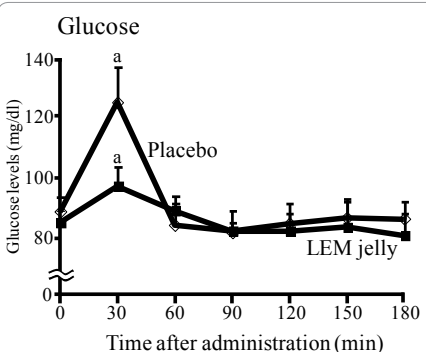

$$
\text { Insulin }
$$

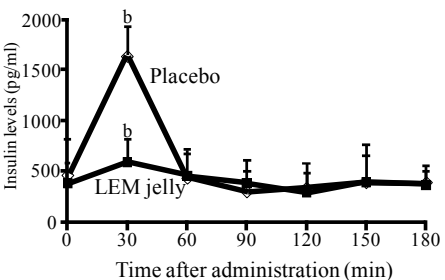

Figure 3: Suppressive effects of LEM jelly on blood glucose and insulin levels in healthy subjects. Data were expressed as means $\pm S D(n=10)$. a, b: There were significant differences in postprandial glucose and insulin responses between healthy subjects ingesting LEM jelly and placebo jelly; $p<0.05$ by paired Student's $t$-test.

suppressed at 30 min after ingestion $(p<0.05)$. The area under the blood glucose curve over $2 \mathrm{~h}$ was $1,106 \pm 321$ after placebo ingestion and 513 \pm 182 after LEM jelly $(p<0.05)$.

\section{Results of hydrogen breath tests after the ingestion of jellies in healthy subjects}

The profiles of hydrogen gas excretion in breath are shown in Figure 4. No hydrogen gas was detected following ingestion of placebo jelly, while hydrogen gas was excreted after the ingestion of LEM jelly, as a result of the inhibition of disaccharidases by LEM. 
Citation: Nakamura S, Hashiguchi M, Yamaguchi Y, Oku T (2011) Hypoglycemic Effects of Morus alba Leaf Extract on Postprandial Glucose and Insulin Levels in Patients with Type 2 Diabetes Treated with Sulfonylurea Hypoglycemic Agents. J Diabetes Metab 2:158. doi:10.4172/21556156.1000158

Page 4 of 5

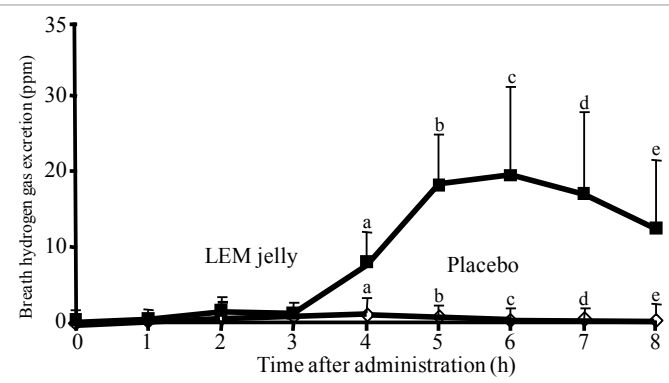

Figure 4: Effects of LEM jelly on breath hydrogen gas excretion in healthy subjects. Data were expressed as means \pm SD $(n=10)$. a-e: There were significant differences in breath hydrogen gas excretion between healthy subjects ingesting LEM and placebo jellies; $p<0.05$ by paired Student's $t$-test.

\section{Discussion}

This study clearly demonstrated that postprandial elevations in glucose and insulin levels in type 2 diabetic patients were significantly suppressed by the ingestion of jelly containing LEM, irrespective of the use of SU. LEM jelly also suppressed postprandial glucose and insulin in healthy subjects. The suppressive effect was significant, despite the range of insulin responses was wide among the patients. Furthermore, LEM jelly caused a marked increase in the excretion of breath hydrogen, indicating a lower energy content, and therefore a likely prebiotic effect.

It is crucial for patients with type 2 diabetes mellitus and highrisk individuals to control their eating behaviors in order to prevent the disease developing to a more serious stage. Jelly containing LEM may help this group to maintain a better lifestyle. The current study examined the interactions between medicine and foods, including a-glucosidase inhibitors. The range of insulin levels in the diabetic patients was wide; however, the suppressive effects of LEM jelly on blood glucose and insulin were observed in patients both with and without SU treatment. One of the reason why the responses of insulin in patients varied from patients to patients is that it was dependent on the ability of the secretion of insulin and the term of the treatment by SU.

This represents important information for patients with type 2 diabetes. It is thought that Japanese patients with type 2 diabetes mellitus tend to show a decline in insulin secretion, rather than increased insulin resistance, resulting in a decrease in the early postprandial secretion of insulin $[33,34]$. LEM may prevent from the failure of beta-cell because it does not stimulate the insulin secretion by the ingestion of available carbohydrate and protected the vascular system against the oxidative stress of the higher concentration of the blood glucose level. Suppression of not only postprandial glucose, but also early postprandial insulin is necessary to prevent the development of complications to a serious stage. Blood glucose and insulin levels are also important for preventing oxidative stress in intravascular endothelial cells, and atherosclerosis. LEM has demonstrated a suppressive effect on atherosclerotic lesions in mice [7] and the protective effect on vascular system through the decreasing of oxidative stress and anti-glycation [18-20], suggesting that it should form part of the dietary therapy for type 1 and 2 diabetes mellitus, and for other metabolic syndromes.

The suppressive effects of LEM on the elevation of blood glucose are caused by inhibition of intestinal disaccharidases, especially sucrase. The excretion of breath hydrogen gas indicates that the breakdown of sucrose in jelly is inhibited by LEM, resulting in its transfer to the large intestine where it is fermented by intestinal microbes. The mechanism responsible for the suppressive effects of LEM jelly on the elevations of blood glucose and insulin can thus be clearly explained. Furthermore, jelly containing LEM can also help to avoid excessive energy intake by preventing sucrose digestion. These results suggest that the ingestion of LEM may help patients with type 2 diabetes mellitus to maintain better systemic health status.

Adverse events, predominantly gastrointestinal symptoms, have frequently been reported in association with the administration of a-glucosidase inhibitors [23-25], though no side-effects resulted from the ingestion of jelly containing LEM in the current study. Kim et al. reported that LEM suppressed postprandial glucose levels with few side-effects by a-glucosidase inhibitor [37]. However, it is necessary to be aware of the possible induction of transitory osmotic diarrhea, which often occurs in association with the ingestion of large amounts of non-digestible carbohydrate $[35,36]$. A ratio of $1: 10$ was previously determined to be a safe and effective ratio of LEM to sucrose in healthy subjects [8], and the present study demonstrated that this ratio was also suitable for patients with type 2 diabetes. It also indicated that the suppressive effects of the test substance on postprandial glucose and insulin levels seen in healthy subjects could be extended to patients with type 2 diabetes. However, its use should be carefully monitored in patients treated with $\alpha$-glucosidase inhibitors.

\section{Conclusion}

The results of this placebo-controlled study confirmed that postprandial glucose and insulin elevations were markedly suppressed after the ingestion of jelly containing LEM in type 2 diabetic patients treated with SU. The addition of LEM also appeared to reduce the available energy content. These results suggest that LEM could be used in processing foods for dietary therapy in type 2 diabetes.

\section{Acknowledgements}

We wish to thank the patients and healthy subjects who participated in this study, and especially Dr. Tsuyoshi Deguchi, the executive director, and the nurses at Miharadai Hospital in Nagasaki City, Japan. We also thank San Onofre in Togitsu, Nagasaki, for making the jellies, and Toyotama Healthy Food Co., Ltd. for providing the LEM.

\section{References}

1. Oku T, Yamada M, Nakamura M, Sadamori N, Nakamura S, et al. (2006) Inhibitory effects of extractives from leaves of Morus alba on human and rat small intestinal disaccharidase activity. Br J Nutr 95: 933-938.

2. Miyahara C, Miyazawa M, Satoh S, Sasaki A, Mizusaki S, et al. (2004) Inhibitory effects of mulberry leaf extract on postprandial hyperglycemia in normal rats. $J$ Nutr Sci Vitaminol 50: 161-164.

3. Kimura T, Nakagawa K, Kubota H, Kojima Y, Goto Y, et al. (2007) Food-grade mulberry powder enriched with 1-deoxynojirimycine suppresses the elevation of postprandial blood glucose in human. J Agric Food Chem 55: 5869-874.

4. Mudra M, Ercan-Fang N, Zhong L, Furne J, Levitt M (2007) Influence of mulberry leaf extract on the blood glucose and breath hydrogen response to ingestion of $75 \mathrm{~g}$ sucrose by type 2 diabetic and control subjects. Diabetes Care 930: 1272-1274.

5. Tanabe K, Nakamura S, Omagari K, Nakamura S (2011) Repeated ingestion of the leaf extract frm Morus alba reduces inslin resistance in Kk-Ay mice. Nutr Res $31: 848-854$. 
Citation: Nakamura S, Hashiguchi M, Yamaguchi Y, Oku T (2011) Hypoglycemic Effects of Morus alba Leaf Extract on Postprandial Glucose and Insulin Levels in Patients with Type 2 Diabetes Treated with Sulfonylurea Hypoglycemic Agents. J Diabetes Metab 2:158. doi:10.4172/21556156.1000158

6. Liu LK, Lee HJ, Shih YW, Chyau CC, Wang CJ, et al. (2008) Mulberry anthocyanin extracts inhibit LDL oxidation and macrophage-derived foam cell formation induced by oxidative LDL. J Food Sci 73: H113-H121.

7. Enkhmaa B, Shiwaku K, Katsube T, Kitajima K, Anuurad E, et al. (2005) Mulberry (Morus alba L.) leaves and their major flavonol quercetin 3-(6-malonylglucoside) attenuate atherosclerotic lesion development in LDL receptor-deficient mice. J Nutr 135: 729-734.

8. Nakamura M, Nakamura S, Oku T (2009) Suppressive response of confections containing the extractive from leaves of Morus alba on postprandial blood glucose and insulin in healthy human subjects. Nutr \& Metab 6: 29

9. Clement S, Braithwaite SS, Magee MF, Ahmann A, Smith EP, et al. (2004) Management of diabetes and hyperglycemia in hospitals. Diabetes Care 27 553-591.

10. Nathan DM, Buse JB, Davidson MB, Heine RJ, Holman RR, et al. (2006) Management of hyperglycemia in type 2 diabetes. A consensus algorithm for the initiation and adjustment of therapy. Diabetes Care 29: 1963-1972.

11. Gonzalez JS, Peyrot M, McCarl LA, Collins EM, Serpa L, et al. (2008) Depression and diabetes treatment nonadherence: a meta-analysis. Diabetes Care 31: 2398-2403.

12. Icks A, Kruse J, Dragano N, Broecker-Preuss U, Mann K, et al. (2008) Heinz Nixdorf Recall Study Investigator Group. Are symptoms of depression more common in diabetes? Results from Nixdorf Recall study. Diabet Med 25: 1330 1336.

13. Aikens JE, Perkins DW, Piette DJ, Lipton B (2008) Association between depression and concurrent type 2 diabetes outcomes varies by diabetes regimen. Diabet Med 25: 1324-1329.

14. Asano N, Oseki K, Kizu H, Matsui K (1994) N-containing sugars from Morus alba and their glycosidase inhibitory activities. Carbohydr Res 259: 243-255.

15. Kaku K, Rasmussen MF, Clauson P, Seino Y (2010) Improved glycaecemic control with minimal hypoglycaemia and no weight change with the once-daily human glucagon-like peptide-1 analogue liraglutide as add-on to sulphonylurea in Japanese patients with type 2 diabetes. Diabetes Dbes Metab 12: 341-347.

16. Nyback-Nakell A, Bergstrom J, Adamson U, Lins PE, Landstedt-Hallin L et al (2010) Decreasing postprandial C-peptide levels over time are not associated with long-term use of sulphonylureaa: an observational study. Diabetes Metab 36: $375-380$

17. Maruyama T, Tanaka S, Shimada A, Funae O, Kasuga A, et al. (2008) Insulin intervention in slowly progressive insulin-dependent (type 1) diabetes mellitus. J Clin Endoclinol Metab 93: 2115-2121.

18. Naowaboot J, Pannangpetch $\mathrm{P}$, Kukongviriyapan V, Kongyingyoes $B$ Kukongviriyapan U, et al. (2009) Antihyperglycemic, antioxidant and antiglycation activities of mulberry leaf extract in streptozotocin-induced chronic diabetic rats. Plant Foods Hum Nutr 64: 116-121.

19. Lee YJ, Cjoi DH, Kim EJ, Kim HY, Kwon TO, et al. (2011) Hypotensive hypolipidemic, and vascular protective effect of Morus alba $L$. in rats fed an atherogenic diet. Am J Clin Med 39: 39-52.

20. El-Sayyad HI, El-Sherbiny MA, Sobn MA, Abou-El-Naga AM, Ibrahim MAN, et al. (2011) Protective effects of Morus alba leaves extract on ocular functions of pups from diabetic and hypercholesterolemic mother rats. Int J Biol Sci 7 : 715-728.

21. Brighenti F, Benini L, Del Rio D, Casiraghi C, Pellegrini N, et al. (2006) Colonic fermentation of indigestible carbohydrates contributes to the second-meal effect. Am J Clin Nutr 83: 817-822.

22. Cummings GH, Macfarlane GT (1991) The control and consequences of bacterial fermentation in the human colon. J Appl Bacteriol 70: 443-459.

23. Sobajima H, Mori M, Niwa T, Muramatsu M, Sugimoto $Y$, et al. (1998) Carbohydrate malabsorption following acarbose administration. Diabet Med 15: 393-397.

24. Drent ML, Tollefsen ATM, van Heusden FHJA, Hoenderdos EBM, Jonker JJC et al. (2002) Dose-dependent efficacy of miglitol, an a-glucosidase inhibitor, in type 2 diabetic patients on diet alone: Results of a 24-week double-blind placebo-controlled study. Diab Nutr Metab 15: 152-159.
25. Holt PR, Atillasoy E, Lindenbaum J, Ho SB, Lupton JR, et al. (1996) Effects of acarbose on fecal nutrients, colonic $\mathrm{pH}$, and short-chain fatty acids and rectal proliferative indices. Metabolism 45: 1179-1187.

26. Oku T, Nakamura S (2002) Digestion, absorption, fermentation, and metabolism of functional sugar substitute and their available energy. Pure Appl Chem 74 1253-1261.

27. Oku T (2005) Digestion, fermentation, absorption and metabolism of nondigestible and/or non-absorbable saccharides and their maximum permissive dosage to produce transitory diarrhea. J Jpn Soc Nutr Food Sci 58: 337-342.

28. Miyazawa M, Miyahara T, Sato S, Sasaki A (2003) Ninety-day dietary toxicity study of mulberry leaf extract in rats. Jpn J Food Hyg 44: 191-197.

29. Oku T, Nakamura M, Takasugi A, Hashiguchi-Ishiguro M, Tanabe K, et al (2009) Effects of cake made from whole soy powder on postprandial blood glucose and insulin levels in human subjects. Int J Food Sci and Nutr 60: 224 231.

30. Nakamura S, Oku T, Ichinose M (2004) Bioavailability of cellobiose by tolerance test and breath hydrogen excretion in humans. Nutrition 20: 979-983.

31. Trinder $P$ (1969) Determination of blood glucose using an oxidase-peroxidase system with a non-carcinogenic chromogen. J Clin Pathol 22: 158-161.

32. Liversy JH, Hodgkinson SC, Round HR, Donald RA (1980) Effect of time temperature and freezing on the stability of immunoreactive $\mathrm{LH}, \mathrm{FSH}, \mathrm{TSH}$ growth hormone, prolactin and insulin in plasma. Clin Biochem 13: 151-155.

33. Shichiri M, Kishikawa H, Ohkubo $Y$, Wake N (2000) Long-term results of the Kumamoto Study on optimal diabetes control in type 2 diabetic patients. Diabetes Care: 23: B21-B29.

34. Araki E, Kishikawa H (2010) Kumamoto Study. Nihon Rinsho 68: 114-120.

35. Oku T, Nakamura S, Ichinose M (2005) Maximum permissive dosage of lactose and lactitol for transitory diarrhea, and utilizable capacity for lactose in Japanese female adults. J Nutr Sci Vitaminol 51: 51-57.

36. Oku T, Nakamura S (2006) Threshold for transitory diarrhea induced by the ingestion of xylitol and lactitol in young male and female adults. J Nutr Sci Vitaminol 53: 13-20.

37. Kim GN, Kwon YI, Jang HD (2011) Mulberry leaf extract reduces postprandia hyperglycemia with few side effects by inhibiting a-glucosidase in normal rats. J Med Food 14: 712-717. 